\title{
Chiral Photomagnets Based on Copper(II) complexes of 1,2-Diaminocyclohexane and Octacyanidomolybdate(IV) ions
}

\author{
Tomasz Korzeniak ${ }^{1 *}$, Sujit Sasmal ${ }^{1}$, Dawid Pinkowicz ${ }^{1}$, Wojciech Nitek ${ }^{1}$, Robert Pelka ${ }^{2}$, \\ Dominik Czernia ${ }^{2}$, Olaf Stefańczyk ${ }^{3}$, Barbara Sieklucka ${ }^{1}$ \\ ${ }^{1}$ Faculty of Chemistry, Jagiellonian University, Gronostajowa 2, 30-387 Kraków, POLAND. \\ ${ }^{2}$ Institute of Nuclear Physics Polish Academy of Sciences, 31-342 Kraków, POLAND. \\ ${ }^{3}$ Department of Chemistry, School of Science, The University of Tokyo, 7-3-1 Hongo, Bunkyo-ku, Tokyo 113-0033, JAPAN.
}

\section{Table of Contents:}

Figure S1. The asymmetric unit of $1 \mathbf{R}$ with selected atoms labeling

The description of asymmetric unit of $\mathbf{1 R}$

Table S1. The values of continuous shape measures (CShM) parameters for $1 \mathbf{R}$ and $\mathbf{1 S}$

The description of SHAPE analysis of coordination geometries

The description of the magnetic data fitting procedure 


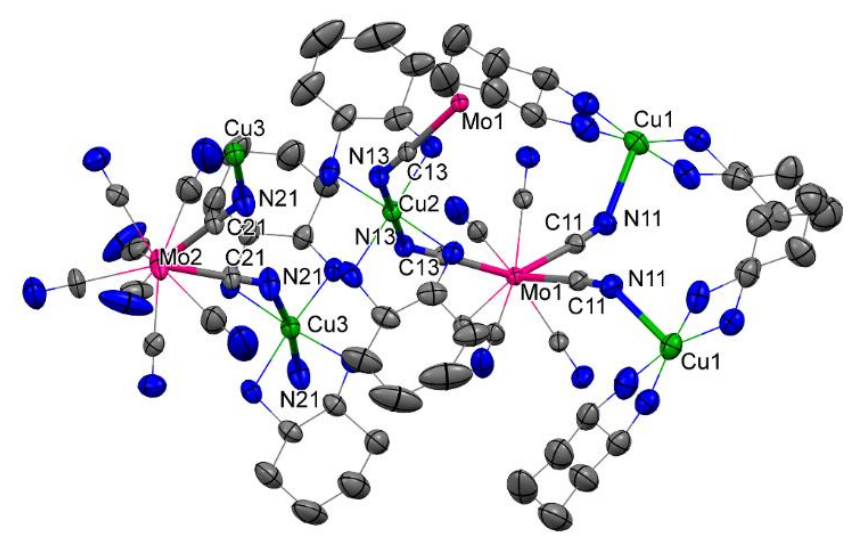

Figure S1. The asymmetric unit of $\mathbf{1 R}$ with selected atoms labeling. The thermal ellipsoids at 30\% probability are shown. Colors used: $\mathrm{Mo}$ - magenta, $\mathrm{Cu}$ - green, $\mathrm{N}$ - blue, $\mathrm{C}$ - grey.

The asymmetric unit is composed of two distinct fragments of the cyanido bridged chains and is presented in Fig S1. The half of the $\left[\mathrm{Mo} 1(\mathrm{CN})_{8}\right]^{4-}$ ion is connected with the $\left[\mathrm{Cu} 1(\mathrm{chxn})_{2}\right]^{2+}$ and half of the $\left[\mathrm{Cu} 2(\mathrm{chxn})_{2}\right]^{2+}$ unit, leading to formation of the helical $\left\{\mathrm{Cu}_{3} \mathrm{Mo}\right\}_{\mathrm{n}}{ }^{2 \mathrm{n}+}$ chain. The planar chain of $\{\mathrm{CuMo}\}_{\mathrm{n}}{ }^{2 \mathrm{n}-}$ formula is reproduced from the fragment consisting of half of $\left[\mathrm{Mo} 2(\mathrm{CN})_{8}\right]^{4-}$ unit bridged towards the half of $\left[\mathrm{Cu} 3(\mathrm{chxn})_{2}\right]^{2+}$ unit. The asymmetric unit is completed by the atoms representing the disordered water molecules with occupancy factors of 0.5 due to disorder.

\section{The SHAPE analysis of coordination geometries}

The SHAPE analysis of the coordination geometries of statistically disordered $\left[\mathrm{Mo} 2(\mathrm{CN})_{8}\right]$ moieties faced problems as the experimentally found structures reveal electron density around Mo2 centers suggesting the presence of nine cyanido ligands. The real structures of $\left[\mathrm{Mo} 2(\mathrm{CN})_{8}\right]$ can be approximated by the removal of one cyanido ligand from nine-coordinated Mo2 coordination environment. Thus the results of analysis for Mo2 take into account the contributions obtained by deleting one of the ligands as described in the table, e. g. "1R Mo2_C25" describes the SHAPE measures for Mo2 in the system 1R with C25-N25 ligand removed.

Table S1. The values of continuous shape measures (CShM) parameters for $\mathbf{1 R}$ and $\mathbf{1 S}$.

$\begin{array}{lccc}\text { Metal center } & \text { SAPR-8 } & \text { TDD-8 } & \text { BTPR-8 } \\ \text { 1R Mo1 } & \mathbf{0 . 2 7 5} & 2.222 & 2.394 \\ \text { 1S Mo1 } & \mathbf{0 . 2 9 1} & 2.306 & 2.447 \\ \text { 1R Mo2_C25 } & 8.074 & 7.101 & \mathbf{4 . 0 6 1} \\ \text { 1R Mo2_C24 } & 3.868 & 3.459 & \mathbf{0 . 7 5 9} \\ \text { 1R Mo2_C23 } & 5.993 & 5.257 & \mathbf{3 . 2 1 1} \\ \text { 1R Mo2_C22 } & 1.695 & 2.032 & \mathbf{0 . 5 4 7} \\ \text { 1R Mo2_C21 } & 8.812 & 7.749 & \mathbf{5 . 6 6 3} \\ \text { 1S Mo2_C25 } & 8.173 & 7.203 & \mathbf{4 . 1 3} \\ \text { 1S Mo2_C24 } & 3.959 & 3.495 & \mathbf{0 . 8 4 1} \\ \text { 1S Mo2_C23 } & 6.024 & 5.245 & \mathbf{3 . 2 3 4} \\ \text { 1S Mo2_C22 } & 1.718 & 2.042 & \mathbf{0 . 5 9 1} \\ \text { 1S Mo2_C21 } & 8.971 & 7.891 & \mathbf{5 . 8 6 6}\end{array}$




\section{The description of magnetic data fitting procedure}

1. Models for the systems before irradiation (Model 0)

1.1. Chiral systems $1 \mathrm{R}$ and $1 \mathrm{~S}$ before irradiation (Model 0 )

1.2. Achiral system 1rac before irradiation (Model 0)

2.Magnetic properties of the systems before irradiation (Model 0)

2.1 Magnetic properties of chiral systems before irradiation

2.2. Magnetic properties of achiral system (1 rac) before irradiation

3. Magnetic properties of the systems after irradiation

3.1. Models of the enantiopure systems after irradiation (Model 1.0 and 1.1)

3.2. Models of the racemic system after irradiation (Model 1.0 and 1.1)

3.3. The simulation of the photomagnetic effect

1. Models for the systems before irradiation (Model 0)

The models used to analyze the magnetic properties of the samples before irradiation involves correlated spin chains (1R or 1S) or honeycomb arrangement (1rac) and exact quantifications of these models would require non-trivial and timeconsuming Monte Carlo simulations, we therefore resort to a simplified approach based on the molecular field approximation (MFA). The fitting of magnetic and photomagnetic properties was performed in a specially designed notebook of the Mathematica8.0 environment.

\subsection{Chiral systems $1 R$ and $1 S$ before irradiation (Model 0$)$}

The chiral systems $\mathbf{1 R}$ and $\mathbf{1 S}$ reveal two cyanido bridged chains of different $\mathrm{Cu}$ : Mo stoichiometry, namely $1: 1$ in the case of alternating Chain 1 and 3:1 in decorated Chain 2 as presented in Scheme S1 (a).

(a) chain 1: $\mathrm{Mo}^{\mathrm{N}} \cdot \mathrm{Cu}^{\prime \prime}$

chain 2: $\mathrm{Mo}^{\mathrm{N}} \cdot \mathrm{Cu} 1^{\prime \prime} \cdot \mathrm{Cu} 2^{\prime \prime}$

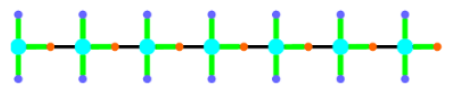

(b)

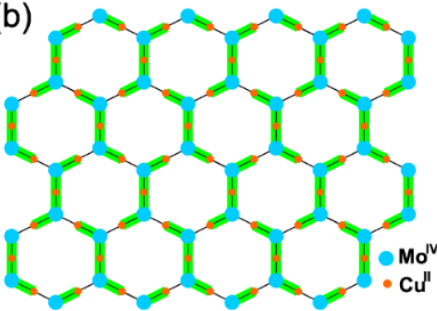

Scheme S1. Model 0 for chiral systems (a) and achiral (b)

The chain 1 consists of two sublattices $\mathrm{A}$ and $\mathrm{B}$ built of $\mathrm{Cu}^{\mathrm{II}}\left(\mathrm{S}_{\mathrm{A}}=1 / 2\right)$ and $\mathrm{Mo}^{\mathrm{IV}}\left(\mathrm{S}_{\mathrm{B}}=0\right)$ ions, revealing $g$ factors of $g_{\mathrm{A}}$ and $g_{\mathrm{B}}$, respectively. Taking into account the stoichiometric factors $v_{\mathrm{A}}$ and $v_{\mathrm{B}}$, the system may be described by the formula $\mathrm{A}_{v \mathrm{~A}} \mathrm{~B}_{\mathrm{v}}$ and the Hamiltonian pertinent to the systems being analyzed is given by the formula: 
$\widehat{H}=-J_{\mathrm{AB}} \sum_{(i j)} \hat{S}_{A i} \cdot \hat{S}_{B j}-J_{\mathrm{AA}} \sum_{(i j)} \hat{S}_{A i} \cdot \hat{S}_{A j}+\mu_{B} \sum_{i}\left(g_{A} \hat{S}_{A i}+g_{B} \hat{S}_{B i}\right) \cdot \vec{H}$

where (ij) denotes the summation over the pairs of the nearest neighbors, $\mathrm{J}_{\mathrm{XY}}$ is superexchange coupling constant between the $\mathrm{X}$ and $\mathrm{Y}$ type ions, $\mu_{\mathrm{B}}$ is the Bohr magneton and $\mathrm{H}$ is the external magnetic field. Let us note that the superexchange coupling within the $\mathrm{B}$ sublattice is neglected $\left(J_{\mathrm{BB}}=0\right)$. The Hamiltonian can be reduced to the form:

$\widehat{H}=g_{A} \mu_{B} \hat{S}_{A} \cdot \vec{H}_{A}+g_{B} \mu_{B} \hat{S}_{B} \cdot \vec{H}_{B}$

in the framework of molecular field approximation. The molecular fields $\mathrm{H}_{\mathrm{A}}$ and $\mathrm{H}_{\mathrm{B}}$ read:

$\vec{H}_{A}=\vec{H}+\Lambda_{\mathrm{AB}} \vec{M}_{B}+\Lambda_{\mathrm{AA}} \vec{M}_{A}$

$\vec{H}_{B}=\vec{H}+\Lambda_{\mathrm{BA}} \vec{M}_{A}$

Quantity $\mathrm{M}_{\mathrm{X}}$ denotes the molar magnetization of sublattice $\mathrm{X}$ and the molecular field constants $\Lambda_{\mathrm{XY}}$ read

$\Lambda_{\mathrm{AB}}=\frac{J_{\mathrm{AB}} Z_{\mathrm{AB}}}{N_{A} \mu_{B}^{2} \nu_{B} g_{A} g_{B}}, \quad \Lambda_{\mathrm{BA}}=\frac{J_{\mathrm{AB}} Z_{\mathrm{BA}}}{N_{A} \mu_{B}^{2} v_{A} g_{A} g_{B}}, \quad \Lambda_{\mathrm{AA}}=\frac{J_{\mathrm{AA}} Z_{\mathrm{AA}}}{N_{A} \mu_{B}^{2} v_{A} g_{A}^{2}}$,

where $\mathrm{Z}_{\mathrm{XY}}$ denotes the number of the nearest neighbor $\mathrm{Y}$ type ions of the $\mathrm{X}$ type ion. As the number of coupling connections between the $\mathrm{A}$ and $\mathrm{B}$ type ions in a mole of the compound can be written as $N_{\mathrm{A}} v_{\mathrm{A}} Z_{\mathrm{AB}}$ or $N_{\mathrm{A}} v_{\mathrm{B}} Z_{\mathrm{BA}}$, hence $v_{\mathrm{A}} Z_{\mathrm{AB}}=v_{\mathrm{B}} Z_{\mathrm{BA}}$. This leads to the only two independent molecular field constants $\Lambda_{\mathrm{AA}}$ and $\Lambda_{\mathrm{AB}}=\Lambda_{\mathrm{AB}} \equiv \Lambda$. For an arbitrary thermodynamic point $(\mathrm{H}, \mathrm{T})$ the molecular field model should be solved by an iterative numerical method. However, in the special case when the temperature is high compared to $\mathrm{J}_{\mathrm{AB}} / \mathrm{k}_{\mathrm{B}}$ and $\mathrm{J}_{\mathrm{AA}} / \mathrm{k}_{\mathrm{B}}$, the system is in the paramagnetic state and the molar magnetization of the sublattice $\mathrm{X}$ is directly proportional to the magnetic field $M_{\mathrm{X}}=\chi_{\mathrm{X}} H_{\mathrm{X}}$, where $\chi_{\mathrm{X}}$ is the paramagnetic molar susceptibility of the sublattice $\mathrm{X}$.

$\chi_{X}=\frac{N_{A} \mu_{B}^{2} v_{X} g_{X}^{2} S_{\mathrm{X} 0}\left(S_{\mathrm{X} 0}+1\right)}{3 k_{B} T}$

The system of linear equations $M_{\mathrm{X}}=\chi_{\mathrm{X}} H_{\mathrm{X}}(\mathrm{X}=\mathrm{A}, \mathrm{B})$ can be solved by the Cramer method for $M_{\mathrm{X}}$ and the total susceptibility of the chain can be calculated as $\chi=\left(\mathrm{M}_{\mathrm{A}}+\mathrm{M}_{\mathrm{B}}\right) / \mathrm{H}$ :

$\chi=\frac{\chi_{A}+\chi_{B}+\chi_{A} \chi_{B}\left(2 \Lambda-\Lambda_{\mathrm{AA}}\right)}{1-\chi_{A} \Lambda_{\mathrm{AA}}-\chi_{A} \chi_{B} \Lambda^{2}}$

The total magnetization of the chain 1 is given by the formula:

$M_{\text {total }}=N_{A} \mu_{B}\left[v_{A} g_{A}\left\langle S_{A}\right\rangle+v_{B} g_{B}\left\langle S_{B}\right\rangle\right]$

where $<\mathrm{S}_{\mathrm{A}}>$ and $<\mathrm{S}_{\mathrm{B}}>$ are the thermally averaged values of sublattice spins:

$\left\langle S_{A}\right\rangle=S_{\mathrm{A} 0} B_{S_{\mathrm{A} 0}}\left[\frac{g_{A} \mu_{B} S_{\mathrm{A} 0} H}{k_{B} T}+\frac{J_{\mathrm{AB}} Z_{\mathrm{AB}} S_{\mathrm{A} 0}}{k_{B} T}\left\langle S_{B}\right\rangle+\frac{J_{\mathrm{AA}} Z_{\mathrm{AA}} S_{\mathrm{A} 0}}{k_{B} T}\left\langle S_{A}\right\rangle\right]$,

$\left\langle S_{B}\right\rangle=S_{\mathrm{B} 0} B_{S_{\mathrm{B} 0}}\left[\frac{g_{B} \mu_{B} S_{\mathrm{B} 0} H}{k_{B} T}+\frac{J_{\mathrm{AB}} Z_{\mathrm{BA}} S_{\mathrm{B} 0}}{k_{B} T}\left\langle S_{A}\right\rangle\right]$,

where $B_{\mathrm{S}}$ is the Brillouin function, $S_{\mathrm{A} 0}$ and $S_{\mathrm{B} 0}$ are the values of $<\mathrm{S}_{\mathrm{A}}>$ and $<\mathrm{S}_{\mathrm{B}}>$ at $0 \mathrm{~K}$, respectively.

The chain 2 consists of three sublattices, namely $\mathrm{A} 1, \mathrm{~A} 2$ built of $\mathrm{Cu}^{\mathrm{II}}$ ions and $\mathrm{B}\left(\mathrm{Mo}^{\mathrm{IV}}\right)$ ions. Taking into account the stoichiometric factors $v_{\mathrm{A} 1}, v_{\mathrm{A} 2}$ and $v_{\mathrm{B}}$ of the respective ions, the system can be described by the formula $\mathrm{A} 1_{\mathrm{vA} 1} \mathrm{~A} 2_{\mathrm{vA} 2} \mathrm{~B}_{\mathrm{vB}}$ and the Hamiltonian pertinent to the system is given by the formula:

$$
\begin{aligned}
\widehat{H}= & -J_{\mathrm{AB}} \sum_{(i j)}\left(\hat{S}_{\mathrm{A} 1 i} \cdot \hat{S}_{B j}+\hat{S}_{\mathrm{A} 2 i} \cdot \hat{S}_{B j}\right)-J_{\mathrm{AA}} \sum_{(i j)}\left(\hat{S}_{\mathrm{A} 1 i} \cdot \hat{S}_{\mathrm{A} 2 j}+\hat{S}_{\mathrm{A} 1 i} \cdot \hat{S}_{\mathrm{A} 1 j}+\hat{S}_{\mathrm{A} 2 i} \cdot \hat{S}_{\mathrm{A} 2 j}\right) \\
& +\mu_{B} \sum_{i}\left[g_{A}\left(\hat{S}_{\mathrm{A} 1 i}+\hat{S}_{\mathrm{A} 2 i}\right)+g_{B} \hat{S}_{B i}\right] \cdot \vec{H}
\end{aligned}
$$


which can be reduced in to the form

$\widehat{H}=g_{A} \mu_{B} \hat{S}_{\mathrm{A} 1} \cdot \vec{H}_{\mathrm{A} 1}+g_{A} \mu_{B} \hat{S}_{\mathrm{A} 2} \cdot \vec{H}_{\mathrm{A} 2}+g_{B} \mu_{B} \hat{S}_{B} \cdot \vec{H}_{B}$

in the framework of molecular field approximation. The molecular fields $\mathrm{H}_{\mathrm{A} 1}, \mathrm{H}_{\mathrm{A} 2}$ and $\mathrm{H}_{\mathrm{B}}$ read:

$\vec{H}_{\mathrm{A} 1}=\vec{H}+\Lambda_{\mathrm{A} 1 \mathrm{~B}} \vec{M}_{B}+\Lambda_{\mathrm{A} 1 \mathrm{~A} 1} \vec{M}_{\mathrm{A} 1}+\Lambda_{\mathrm{A} 1 \mathrm{~A} 2} \vec{M}_{\mathrm{A} 2}$

$\vec{H}_{\mathrm{A} 2}=\vec{H}+\Lambda_{\mathrm{A} 2 \mathrm{~B}} \vec{M}_{B}+\Lambda_{\mathrm{A} 2 \mathrm{~A} 1} \vec{M}_{\mathrm{A} 1}+\Lambda_{\mathrm{A} 2 \mathrm{~A} 2} \vec{M}_{\mathrm{A} 2}$

$\vec{H}_{B}=\vec{H}+\Lambda_{\mathrm{BA} 1} \vec{M}_{\mathrm{A} 1}+\Lambda_{\mathrm{BA} 2} \vec{M}_{\mathrm{A} 2}$

Quantity $\mathrm{M}_{\mathrm{X}}$ denotes the molar magnetization of sublattice $\mathrm{X}$ and the molecular filed constants are given by:

$\Lambda_{\mathrm{A} 1 \mathrm{~B}}=\frac{J_{\mathrm{AB}} Z_{\mathrm{A} 1 \mathrm{~B}}}{N_{A} \mu_{B}^{2} v_{B} g_{A} g_{B}}, \quad \Lambda_{\mathrm{BA} 1}=\frac{J_{\mathrm{AB}} Z_{\mathrm{BA} 1}}{N_{A} \mu_{B}^{2} v_{\mathrm{A} 1} g_{A} g_{B}}$,

$\Lambda_{\mathrm{A} 2 \mathrm{~B}}=\frac{J_{\mathrm{AB}} Z_{\mathrm{A} 2 \mathrm{~B}}}{N_{A} \mu_{B}^{2} v_{B} g_{A} g_{B}}, \quad \Lambda_{\mathrm{BA} 2}=\frac{J_{\mathrm{AB}} Z_{\mathrm{BA} 2}}{N_{A} \mu_{B}^{2} v_{\mathrm{A} 2} g_{A} g_{B}}$,

$\Lambda_{\mathrm{A} 1 \mathrm{~A} 2}=\frac{J_{\mathrm{AA}} Z_{\mathrm{A} 1 \mathrm{~A} 2}}{N_{A} \mu_{B}^{2} v_{\mathrm{A} 2} g_{A}^{2}}, \quad \Lambda_{\mathrm{A} 2 \mathrm{~A} 1}=\frac{J_{\mathrm{AA}} Z_{\mathrm{A} 2 \mathrm{~A} 1}}{N_{A} \mu_{B}^{2} v_{\mathrm{A} 1} g_{A}^{2}}$,

$\Lambda_{\mathrm{A} 1 \mathrm{~A} 1}=\frac{J_{\mathrm{AA}} Z_{\mathrm{A} 1 \mathrm{~A} 1}}{N_{A} \mu_{B}^{2} v_{\mathrm{A} 1} g_{A}^{2}}, \quad \Lambda_{\mathrm{A} 2 \mathrm{~A} 2}=\frac{J_{\mathrm{AA}} Z_{\mathrm{A} 2 \mathrm{~A} 2}}{N_{A} \mu_{B}^{2} v_{\mathrm{A} 2} g_{A}^{2}}$,

where $Z_{X Y}$ denotes the number of the nearest neighbor $\mathrm{Y}$ ions of the $\mathrm{X}$ type ions. The number of molecular field constants can be reduced to five:

$\Lambda_{1} \equiv \Lambda_{\mathrm{A} 1 \mathrm{~B}}=\Lambda_{\mathrm{BA} 1} ; \Lambda_{2} \equiv \Lambda_{\mathrm{A} 2 \mathrm{~B}}=\Lambda_{\mathrm{BA} 2} ; \Lambda_{3} \equiv \Lambda_{\mathrm{A} 1 \mathrm{~A} 2}=\Lambda_{\mathrm{A} 2 \mathrm{~A} 1} ; \Lambda_{4} \equiv \Lambda_{\mathrm{A} 1 \mathrm{~A} 1}$ and $\Lambda_{5} \equiv \Lambda_{\mathrm{A} 2 \mathrm{~A} 2}$

as number of coupling connections between $\mathrm{A} 1$ and $\mathrm{B}$ type ions in a mole of compound can be written either as $N_{\mathrm{A}} v_{\mathrm{A} 1} \mathrm{Z}_{\mathrm{A} 1 \mathrm{~B}}$ or as $N_{\mathrm{A}} v_{\mathrm{B}} Z_{\mathrm{BA}}$, hence $v_{\mathrm{A} 1} Z_{\mathrm{A} 1 \mathrm{~B}}=v_{\mathrm{B}} Z_{\mathrm{BA} 1}$. When the system is in the paramagnetic state with the molar magnetization of the sublattice $\mathrm{X}$ directly proportional to the magnetic field $M_{\mathrm{X}}=\chi_{\mathrm{X}} H_{\mathrm{X}}$, where the molar susceptibility of the sublattice $\mathrm{X}$ is given by the formula $\chi_{X}=\frac{N_{A} \mu_{B}^{2} v_{X} g_{X}^{2} S_{\mathrm{X} 0}\left(S_{\mathrm{X} 0}+1\right)}{3 k_{B} T}$

The system of linear equations $M_{\mathrm{X}}=\chi_{\mathrm{X}} H_{\mathrm{X}}(\mathrm{X}=\mathrm{A} 1, \mathrm{~A} 2, \mathrm{~B})$ can be solved by the Cramer method for $\mathrm{M}_{\mathrm{X}}$ an the total susceptibility of the second chain is given by:

$\chi=\frac{M_{\mathrm{A} 1}+M_{\mathrm{A} 2}+M_{B}}{H} \equiv \frac{N}{D}$

where

$$
\begin{array}{r}
N=\chi_{\mathrm{A} 1}+\chi_{\mathrm{A} 1}+\chi_{B}+\chi_{\mathrm{A} 1} \chi_{B}\left(2 \Lambda_{1}-\Lambda_{4}\right)+\chi_{\mathrm{A} 2} \chi_{B}\left(2 \Lambda_{2}-\Lambda_{5}\right)+\chi_{\mathrm{A} 1} \chi_{\mathrm{A} 2}\left(2 \Lambda_{3}-\Lambda_{4}-\Lambda_{5}\right) \\
-\chi_{\mathrm{A} 1} \chi_{\mathrm{A} 2} \chi_{B}\left(\Lambda_{1}^{2}+\Lambda_{2}^{2}+\Lambda_{3}^{2}-2 \Lambda_{1} \Lambda_{2}-2 \Lambda_{1} \Lambda_{3}-2 \Lambda_{2} \Lambda_{3}+2 \Lambda_{1} \Lambda_{5}+2 \Lambda_{2} \Lambda_{4}-\Lambda_{4} \Lambda_{5}\right)
\end{array}
$$

and

$$
\begin{aligned}
D & =1-\chi_{\mathrm{A} 1} \Lambda_{4}-\chi_{\mathrm{A} 2} \Lambda_{5}-\chi_{\mathrm{A} 1} \chi_{B} \Lambda_{1}^{2}-\chi_{\mathrm{A} 2} \chi_{B} \Lambda_{2}^{2}-\chi_{\mathrm{A} 1} \chi_{\mathrm{A} 2}\left(\Lambda_{3}^{2}-\Lambda_{4} \Lambda_{5}\right) \\
& +\chi_{\mathrm{A} 1} \chi_{\mathrm{A} 2} \chi_{B}\left(\Lambda_{1}^{2} \Lambda_{5}+\Lambda_{2}^{2} \Lambda_{4}-2 \Lambda_{1} \Lambda_{2} \Lambda_{3}\right)
\end{aligned}
$$

To compute the total molar magnetization of chain 2

$M_{\text {total }}=N_{A} \mu_{B}\left[v_{\mathrm{A} 1} g_{A}\left\langle S_{\mathrm{A} 1}\right\rangle+v_{\mathrm{A} 2} g_{A}\left\langle S_{\mathrm{A} 2}\right\rangle+v_{B} g_{B}\left\langle S_{B}\right\rangle\right]$

where $\left\langle\mathrm{S}_{\mathrm{X}}\right\rangle$ are the thermally averaged values of sublattice spins, that needs to be solved iteratively from a set of coupled equations: 


$$
\begin{aligned}
& \left\langle S_{\mathrm{A} 1}\right\rangle=S_{\mathrm{A} 0} B_{S_{\mathrm{A} 0}}\left[\frac{g_{A} \mu_{B} S_{\mathrm{A} 0} H}{k_{B} T}+\frac{J_{\mathrm{AB}} Z_{\mathrm{A} 1 \mathrm{~B}} S_{\mathrm{A} 0}}{k_{B} T}\left\langle S_{B}\right\rangle+\frac{J_{\mathrm{AA}} Z_{\mathrm{A} 1 \mathrm{~A} 1} S_{\mathrm{A} 0}}{k_{B} T}\left\langle S_{\mathrm{A} 1}\right\rangle+\frac{J_{\mathrm{AA}} Z_{\mathrm{A} 1 \mathrm{~A} 2} S_{\mathrm{A} 0}}{k_{B} T}\left\langle S_{\mathrm{A} 2}\right\rangle\right], \\
& \left\langle S_{\mathrm{A} 2}\right\rangle=S_{\mathrm{A} 0} B_{S_{\mathrm{A} 0}}\left[\frac{g_{A} \mu_{B} S_{\mathrm{A} 0} H}{k_{B} T}+\frac{J_{\mathrm{AB}} Z_{\mathrm{A} 2 \mathrm{~B}} S_{\mathrm{A} 0}}{k_{B} T}\left\langle S_{B}\right\rangle+\frac{J_{\mathrm{AA}} Z_{\mathrm{A} 2 \mathrm{~A} 1} S_{\mathrm{A} 0}}{k_{B} T}\left\langle S_{\mathrm{A} 1}\right\rangle+\frac{J_{\mathrm{AA}} Z_{\mathrm{A} 2 \mathrm{~A} 2} S_{\mathrm{A} 0}}{k_{B} T}\left\langle S_{\mathrm{A} 2}\right\rangle\right], \\
& \left\langle S_{B}\right\rangle=S_{\mathrm{B} 0} B_{S_{\mathrm{B} 0}}\left[\frac{g_{B} \mu_{B} S_{\mathrm{B} 0} H}{k_{B} T}+\frac{J_{\mathrm{AB}} Z_{\mathrm{BA} 1} S_{\mathrm{B} 0}}{k_{B} T}\left\langle S_{\mathrm{A} 1}\right\rangle+\frac{J_{\mathrm{AB}} Z_{\mathrm{BA} 2} S_{\mathrm{B} 0}}{k_{B} T}\left\langle S_{\mathrm{A} 2}\right\rangle\right],
\end{aligned}
$$

where $B_{\mathrm{S}}$ is the Brillouin function, $S_{\mathrm{A} 0}$ and $S_{\mathrm{B} 0}$ are the values of $<\mathrm{S}_{\mathrm{A}}>$ and $<\mathrm{S}_{\mathrm{B}}>$ at $0 \mathrm{~K}$, respectively.

\subsection{Achiral system 1rac before irradiation (Model 0)}

The model used for the achiral system is shown in Scheme S1 (b). The hexagonal 2-D layer consists of $\mathrm{Cu}^{\mathrm{II}}$ centers surrounded by diamagnetic $\left[\mathrm{Mo}^{\mathrm{IV}}(\mathrm{CN})_{8}\right]^{4-}$ spacer and each of the octacyanidomolybdate moiety connects two $\mathrm{Cu}^{\mathrm{II}}$ centers. The coupling between $\mathrm{Cu}^{\mathrm{II}}$ centers bridged by the diamagnetic $\left[\mathrm{Mo}^{\mathrm{IV}}(\mathrm{CN})_{8}\right]^{4-}$ units is non-negligible and quantified by the $J_{\mathrm{CuCu}}$ coupling constant. In the case of achiral system the non-bridging $\left[\mathrm{Cu}^{\mathrm{II}}( \pm-\mathrm{chxn})\left(\mathrm{H}_{2} \mathrm{O}\right)_{2}\right]^{2+}$ cations are situated between the layers and reveal very small magnetic interactions with other $\mathrm{Cu}^{\mathrm{II}}$ centers that can be neglected.

Thus the model used to analyze the magnetic properties of 1rac before irradiation (model 0) is the same as used in the modeling of chain 1. In this case sublattice A corresponds to the $\mathrm{Cu}^{\mathrm{II}}$ sublattice and sublattice $\mathrm{B}$ corresponds to $\mathrm{Mo}^{\mathrm{IV}}$ sublattice, forming the $\mathrm{Cu}_{3} \mathrm{Mo}_{2}$ hexagonal layer. The pertinent values of parameters are $v_{\mathrm{Cu}}=3, v_{\mathrm{Mo}}=2, \mathrm{Z}_{\mathrm{CuMo}}=2, \mathrm{Z}_{\mathrm{CuCu}}=4 \mathrm{~S}_{\mathrm{Cu} 0}=1 / 2$ and $\mathrm{S}_{\mathrm{Mo}}=0$.

\section{Magnetic properties of the systems before irradiation (Model 0)}

\subsection{Magnetic properties of chiral systems before irradiation}

The magnetic properties of $\mathbf{1 R}$ and $\mathbf{1 S}$ are similar and reveal almost paramagnetic behavior shown in Fig. 4. as linear plots of $\chi_{M} T$ vs. T above $10 \mathrm{~K}$. The small upshifts are present in the lower temperatures region, which implies non-negligible ferromagnetic interactions. The experimental $\chi_{M} T$ values at $300 \mathrm{~K}$ read 0.868 and $0.849 \mathrm{~cm}^{3} \cdot \mathrm{K} \cdot \mathrm{mol}^{-1}$ for $1 \mathbf{R}$ and $1 \mathrm{~S}$ enantiomers, respectively, which is consistent with the presence of two spins $S=1 / 2$ with $g=2.151$ and 2.128 for $\mathbf{1 R}$ and $\mathbf{1 S}$. The magnetization $v s$ field plots are shown in Figure 5 and reveal typical paramagnetic shape. The values of magnetization in the field of $7 \mathrm{~T}$ show 2.10 and $2.03 N_{\mathrm{A}} \mu_{\mathrm{B}}$, which implies the values of $g$ factor above 2 for $\mathrm{Cu}^{\mathrm{II}}$ centers in both systems. In order to get quantitative insight into the magnetic interactions present in the system before irradiation the model 0 is assumed, where in-chain $\mathrm{Cu}^{\mathrm{II}}$ ions are coupled via the superexchange interactions mediated through the diamagnetic $-\mathrm{NC}-\mathrm{Mo}^{\mathrm{IV}}-\mathrm{CN}-$ linkages. Based on the equations derived for the two chains present in the formula unit (see section 1.1), the magnetic susceptibility of the system was fitted to the experimental data using the formula:

$\chi_{0} T=\frac{1}{2} \frac{N_{A} \mu_{B}^{2} g_{\mathrm{Cu}}^{2}}{3 k_{B}}\left[\frac{\frac{3}{4} g_{\mathrm{Cu}}^{2}}{1-\frac{J_{\mathrm{CuCu}}}{2 k_{B} T}}+\frac{\frac{9}{4} g_{\mathrm{Cu}}^{2}+\frac{9}{16} g_{\mathrm{Cu}}^{2} \frac{J_{\mathrm{CuCu}}}{k_{B} T}}{1-\frac{3}{4} \frac{J_{\mathrm{CuCu}}}{k_{B} T}-\frac{3}{8}\left(\frac{J_{\mathrm{CuCu}}}{k_{B} T}\right)^{2}}\right]$

The results of the fitting procedure yield $J_{\mathrm{CuCu}}=+0.0025(9) \mathrm{cm}^{-1}$ and $g_{\mathrm{Cu}}=2.1510(2)$ for $\mathbf{1 R}$ and $J_{\mathrm{CuCu}}=+0.0131(6) \mathrm{cm}^{-1}$ and $g_{\mathrm{Cu}}=2.12413(3)$ for $\mathbf{1 S}$, which is consistent with the presence of weak ferromagnetic interactions. 


\subsection{Magnetic properties of achiral system (1rac) before irradiation}

The magnetic properties of $1 \mathbf{r a c}$ before irradiation are presented in Figure 4 and reveal almost paramagnetic system. However, a steep downshift in the temperature range below $30 \mathrm{~K}$ indicates the presence of non-negligible antiferromagnetic interactions. The experimental $\chi_{M} T$ value at $300 \mathrm{~K}$ equals $0.861 \mathrm{~cm}^{3} \cdot \mathrm{mol}^{-1} \cdot \mathrm{K}$, which corresponds to the presence of two spins $\mathrm{S}=1 / 2$ and $g=2.14$. The magnetization $v$ field shown in Figure 5 reaches $2.18 \mathrm{~N} \beta$ at $7 \mathrm{~T}$, which is consistent with the presence of two spins $\mathrm{S}=1 / 2$ with $g$ value above 2 . The analysis of the magnetic properties of 1 rac with the use of model 0 and addition of the contribution from the isolated $\left[\mathrm{Cu}^{\mathrm{II}}(\operatorname{chxn})_{2}\left(\mathrm{H}_{2} \mathrm{O}\right)_{2}\right]^{2+}$ ions leads to the following formula for $\chi_{M} T$ :

$\chi_{0} T=\frac{1}{2} \frac{N_{A} \mu_{B}^{2} g_{\mathrm{Cu}}^{2}}{4 k_{B}}\left[\frac{3}{1-J_{\mathrm{CuCu}} / k_{B} T}+\frac{1}{\cosh ^{2}\left(\mu_{B} g_{\mathrm{Cu}} H_{0} / 2 k_{B} T\right)}\right]$

The magnetization can be calculated from the equation:

$M_{0}=\frac{1}{2}\left[M_{\text {total } 0}+\frac{1}{2} N_{A} \mu_{B} g_{\mathrm{Cu}} \tanh \left(\frac{\mu_{B} g_{\mathrm{Cu}} H}{2 k_{B} T}\right)\right]$

where $M_{\text {total } 0}$ denotes the iterative solution of the system in model 0 according to the formula presented in Section 1.1. The fitting procedure of the experimental data yields $J_{\mathrm{CuCu}}=-0.680(3) \mathrm{cm}^{-1}$ and $g=2.1457(1)$, which indicates the presence of weak antiferromagnetic in-layer magnetic coupling. The failure to reproduce faithfully the $\chi_{M} T$ values at the lowest temperature range may be attributed to the fact that MFA cannot correctly account for the geometrical frustration of the triangular sublattice formed by the in-layer $\mathrm{Cu}$ (II) ions. The deviation of the calculated magnetization from the experimental data originates from the wellknown feature of the iterative MFA procedure to underestimate the result in case of relatively large magnetization amplitudes. The set of the best-fit parameters serve as an input for the analysis of the susceptibility signal upon irradiation.

\section{Magnetic properties of the systems after irradiation}

\subsection{Models of the enantiopure systems after irradiation (Model 1.0 and 1.1)}

The description of the photoexcited state of chiral and achiral systems requires the application of the model for excited sample. Taking into account the yield of photoconversion, two different models have been used: diluted model 1.0 for low degree of photomagnetic effect and the saturated model 1.1, which are shown in Scheme S2.

The diluted model 1.0 describes the case of low yield of photomagnetic effect. In this case the chain 1 contains $\mathrm{Cu}_{2}{ }_{2} \mathrm{Mo}^{\mathrm{IV}}{ }_{\mathrm{HS}}$

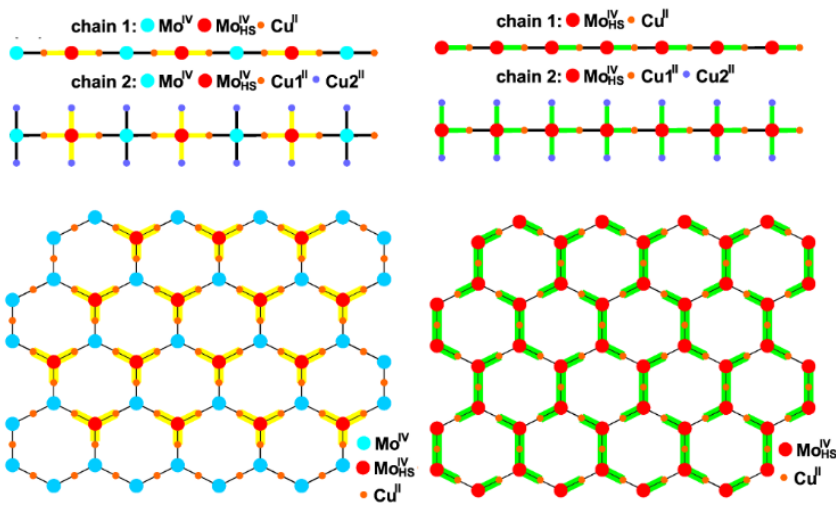

Scheme S2. Model 1.0 (left) and Model 1.1(right) for chiral systems (a) and achiral (b)

isolated clusters and $\mathrm{Cu}^{\mathrm{II}}$ ions mediated by the diamagnetic $\mathrm{Mo}^{\mathrm{IV}}$ LS centers. The chain 2 reveals the presence of $\mathrm{Cu}_{4} \mathrm{MMo}^{\mathrm{IV}} \mathrm{HS}^{\mathrm{IV}}$ accompanied by $\mathrm{Cu}^{\mathrm{II}}$ ions mediated by $\mathrm{Mo}^{\mathrm{IV}}$ diamagnetic spacers. As the halved sum of the contributions from both chains does not reproduce the stoichiometry of the formula unit, the contribution of two $\mathrm{Cu}^{\mathrm{II}}$ centers should be substracted. The molar susceptibility is given by 
$\chi_{f 1.0} T=\frac{1}{2}\left[\chi_{\mathrm{f} 1}\left(H_{0}, T\right) T+\chi_{\mathrm{f} 2}\left(H_{0}, T\right) T-\frac{N_{A} \mu_{B}^{2} g_{\mathrm{Cu}}^{2}}{2 k_{B}} \frac{1}{\cosh ^{2}\left(\mu_{B} g_{\mathrm{Cu}} H_{0} / 2 k_{B} T\right)}\right]$

The cluster contributions $\chi_{\mathrm{fl}}$ and $\chi_{f 2}$ can be obtained by diagonalization of the respective Hamiltonians:

$\widehat{H}_{\mathrm{f} 1}=-J_{\mathrm{CuMo}} \hat{S}_{\mathrm{Mo}} \cdot\left(\hat{S}_{\mathrm{Cu} 1}+\hat{S}_{\mathrm{Cu} 2}\right)+g_{\mathrm{Mo}} \mu_{B} \hat{S}_{\mathrm{Mo}} \cdot \vec{H}+g_{\mathrm{Cu}} \mu_{B}\left(\hat{S}_{\mathrm{Cu} 1}+\hat{S}_{\mathrm{Cu} 2}\right) \cdot \vec{H}$

$\widehat{H}_{\mathrm{f} 2}=-J_{\mathrm{CuMo}} \hat{S}_{\mathrm{Mo}} \cdot\left(\hat{S}_{\mathrm{Cu} 1}+\hat{S}_{\mathrm{Cu} 2}+\hat{S}_{\mathrm{Cu} 3}+\hat{S}_{\mathrm{Cu} 4}\right)+g_{\mathrm{Mo}} \mu_{B} \hat{S}_{\mathrm{Mo}} \cdot \vec{H}+g_{\mathrm{Cu}} \mu_{B}\left(\hat{S}_{\mathrm{Cu} 1}+\hat{S}_{\mathrm{Cu} 2}+\hat{S}_{\mathrm{Cu} 3}+\hat{S}_{\mathrm{Cu} 4}\right) \cdot \vec{H}$

The molar magnetization and the susceptibility have been calculated as appropriate derivates of the partition function:

$M_{L}(H, T)=\frac{N_{A}}{\beta} \frac{\partial \ln Z_{L}(H, T)}{\partial H} \quad \chi_{L}(H, T)=\frac{N_{A}}{\beta} \frac{\partial^{2} \ln Z_{L}(H, T)}{\partial H^{2}}$

where $\beta=1 / k_{\mathrm{B}} \mathrm{T}$, and $\mathrm{L}=\mathrm{f} 1$ or $\mathrm{f} 2$.

The molar magnetization is the sum of the three contributions:

$M_{f 1.0}=\frac{1}{2}\left[M_{\mathrm{f} 1}(H, T)+M_{\mathrm{f} 2}(H, T)-N_{A} \mu_{B} g_{\mathrm{Cu}} \tanh \left(\frac{\mu_{B} g_{\mathrm{Cu}} H}{2 k_{B} T}\right)\right]$

The saturated model (model 1.1) depicts the situation when the molar fraction of $\mathrm{Mo}^{\mathrm{IV}} \mathrm{HS}$ is equal to 1 . Then the magnetic susceptibility is given by the formula:

$\chi_{1.1} T=\frac{1}{2} \frac{N_{A} \mu_{B}^{2} g_{\mathrm{Cu}}^{2}}{3 k_{B}}\left[\begin{array}{l}\frac{\frac{3}{4} g_{\mathrm{Cu}}^{2}+2 g_{\mathrm{Mo}}^{2}+2 g_{\mathrm{Cu}} g_{\mathrm{Mo}} \frac{J_{\mathrm{CuMo}}}{k_{B} T}}{1-\frac{2}{3}\left(\frac{J_{\mathrm{CuMo}}}{k_{B} T}\right)^{2}} \\ +\frac{\frac{9}{4} g_{\mathrm{Cu}}^{2}+2 g_{\mathrm{Mo}}^{2}+4 g_{\mathrm{Cu}} g_{\mathrm{Mo}} \frac{J_{\mathrm{CuMo}}}{k_{B} T}-\frac{1}{4} g_{\mathrm{Cu}}^{2}\left(\frac{J_{\mathrm{CuMo}}}{k_{B} T}\right)^{2}}{1-\left(\frac{J_{\mathrm{CuMo}}}{k_{B} T}\right)^{2}}\end{array}\right]$

where the first contribution originates from the correlated chain 1 and the second contribution corresponds to the correlated chain 2 . The molar magnetization in model 1.1 is given by the formula

$M_{1.1}=\frac{1}{2}\left[M_{\text {total c1 }}+M_{\text {total c2 }}\right]$

where $M_{\text {total c1 }}$ and $M_{\text {total c2 }}$ denote the total magnetization of chains 1 and 2, respectively.

\subsection{Models of the racemic system after irradiation (Model 1.0 and 1.1)}

The diluted model 1.0 Pertinent to the photoinduced configuration with a relatively low molar fraction of the excited $\mathrm{Mo}^{\mathrm{IV}}{ }_{\mathrm{HS}}$ ions we assume model 1.0 shown schematically in Scheme S2 (b, left). The corresponding molar susceptibility is again a sum of two contributions

$\chi_{1.0} T=\frac{1}{2}\left[\chi_{L}\left(H_{0}, T\right) T+\frac{N_{A} \mu_{B}^{2} g_{\mathrm{Cu}}^{2}}{4 k_{B}} \frac{1}{\cosh ^{2}\left(\mu_{B} g_{\mathrm{Cu}} H_{0} / 2 k_{B} T\right)}\right]$.

To obtain the layer contribution $\chi_{\mathrm{L}}(H, T)$ one has to numerically diagonalize the Hamiltonian corresponding to the superexchange-coupled isolated in-layer unit $[\mathrm{Cu}(\mathrm{II})]_{3} \mathrm{Mo}(\mathrm{IV})_{\mathrm{HS}}$ 
$\widehat{H}_{L}=-J_{\mathrm{CuMo}} \hat{S}_{\mathrm{Mo}} \cdot\left(\hat{S}_{\mathrm{Cu} 1}+\hat{S}_{\mathrm{Cu} 2}+\hat{S}_{\mathrm{Cu} 2}\right)+g_{\mathrm{Mo}} \mu_{B} \hat{S}_{\mathrm{Mo}} \cdot \vec{H}+g_{\mathrm{Cu}} \mu_{B}\left(\hat{S}_{\mathrm{Cu} 1}+\hat{S}_{\mathrm{Cu} 2}+\hat{S}_{\mathrm{Cu} 2}\right) \cdot \vec{H}$,

and to calculate the field- and temperature dependent partition function $Z_{\mathrm{L}}(H, T)$. Then the molar magnetization and susceptibility is obtained as appropriate derivatives of the partition function, i.e.

$M_{L}(H, T)=\frac{N_{A}}{\beta} \frac{\partial \ln Z_{L}(H, T)}{\partial H}, \quad \quad \chi_{L}(H, T)=\frac{N_{A}}{\beta} \frac{\partial^{2} \ln Z_{L}(H, T)}{\partial H^{2}}$,

where $\beta=1 / k_{\mathrm{B}} T$. The molar magnetization in this model is likewise a sum of two following contributions

$M_{1.0}=\frac{1}{2}\left[M_{L}(H, T)+\frac{1}{2} N_{A} \mu_{B} g_{\mathrm{Cu}} \tanh \left(\frac{\mu_{B} g_{\mathrm{Cu}} H}{2 k_{B} T}\right)\right]$.

The saturated model 1.1. Pertinent to the photoinduced configuration with the molar fraction of the excited $\mathrm{Mo}^{\mathrm{IV}} \mathrm{HS}_{\text {ions }}$ equal to 1 we assume model 1.1 shown schematically in Scheme S2 (b, right). The corresponding molar susceptibility is again a sum of two contributions

$\chi_{1.1} T=\frac{1}{2} \frac{N_{A} \mu_{B}^{2} g_{\mathrm{Cu}}^{2}}{12 k_{B}}\left[\frac{9 g_{\mathrm{Cu}}^{2}+16 g_{\mathrm{Mo}}^{2}+24 g_{\mathrm{Cu}} g_{\mathrm{Mo}} J_{\mathrm{CuMo}} / k_{B} T}{1-\left(J_{\mathrm{CuMo}} / k_{B} T\right)^{2}}+\frac{3}{\cosh ^{2}\left(\mu_{B} g_{\mathrm{Cu}} H_{0} / 2 k_{B} T\right)}\right]$,

where the first contribution originating from the correlated hexagonal layer was obtained with model for chain 1 in Section 1 assuming $S_{\mathrm{Mo} 0}=1$ and $J_{\mathrm{CuCu}}=0$. The molar magnetization in model 1.1 is given by the formula

$M_{1.1}=\frac{1}{2}\left[M_{\text {total } 1.1}+\frac{1}{2} N_{A} \mu_{B} g_{\mathrm{Cu}} \tanh \left(\frac{\mu_{B} g_{\mathrm{Cu}} H}{2 k_{B} T}\right)\right]$,

where $M_{\text {total } 1.1}$ denotes the iterative solution of the corresponding system of equations for magnetization of chain 1.

\subsection{The simulation of the photomagnetic effect}

The simulation of the photoinduced magnetization needs to take into account the fact that the $\mathrm{Mo}^{\mathrm{IV}}{ }_{\mathrm{HS}}$ is a metastable state and relaxes back upon heating the sample. This can be assumed by the exponential decrease of the molar fraction of $\mathrm{Mo}^{\mathrm{IV}}{ }_{\mathrm{HS}}$ spin, $f_{1}$ :

$f_{1}=f_{10} \exp \left(-\frac{t}{\tau}\right)$

where $f_{10}$ is the initial molar fraction of the photogenerated $\mathrm{Mo}^{\mathrm{IV}} \mathrm{HS}$ centers and $t$ is a mean lifetime of the metastable state. The energy barrier preventing the relaxation to the ground state is denoted by $\Delta$ and related to the mean lifetime by an exponential relation:

$\tau=\tau_{0} \exp \left(\frac{\Delta}{k_{B} T}\right)$

where $\tau_{0}$ defines the high-temperature limit of the lifetime. The total susceptibility of the system upon irradiation is a combination of two contributions: i) the signal of the sample in the excited state with the $f_{1}$ share, and ii) the signal of the sample in its ground state, which has $1-f_{1}$ share. 